\title{
Incoordination during the pharyngeal phase in severe dysphagia due to lateral medullary syndrome
}

Kenjiro Kunieda ${ }^{1}$, Takafumi Sugi ${ }^{2}$, Tomohisa Ohno ${ }^{2}$, Akiko Nomoto ${ }^{2}$, Takashi

Shigematsu $^{2}$, Hideaki Kanazawa ${ }^{2}$, and Ichiro Fujishima ${ }^{2}$

${ }^{1}$ Gifu University Graduate School of Medicine

${ }^{2}$ Hamamatsu City Rehabilitation Hospital

October 8, 2020

Incoordination during the pharyngeal phase in severe dysphagia due to lateral medullary syndrome

Running title: Severe dysphagia due to LMS

Authors:

Kenjiro Kunieda ${ }^{1,2}$, Takafumi $\mathrm{Sugi}^{2}$, Tomohisa Ohno ${ }^{3}$, Akiko Nomoto ${ }^{3}$, Takashi Shigematsu ${ }^{2}$, Hideaki Kanazawa $^{2}$, Ichiro Fujishima ${ }^{2}$

1. Department of Neurology, Gifu University Graduate School of Medicine, Gifu, Japan

2. Department of Rehabilitation Medicine, Hamamatsu City Rehabilitation Hospital, Hamamatsu, Japan

3. Department of Dentistry, Hamamatsu City Rehabilitation Hospital, Hamamatsu, Japan

Funding: None

Conflict of interest: The authors state that they have no conflicts of interest.

Corresponding author:

Kenjiro Kunieda

Department of Neurology, Gifu University Graduate School of Medicine.

1-1 Yanagido, Gifu 501-1194, Japan

Phone: +81582306254.

Fax: $\quad+81582306252$.

E-mail address: ken2rou.k@gmail.com

Acknowledgments: None

Informed consent: This study was approved by the Ethics Committee of our hospital. The patient provided informed consent.

Key words : lateral medullary syndrome, high-resolution manometry, incoordination, central pattern generator, upper esophageal sphincter

Clinical Message: 
One of the mechanisms of severe dysphagia due to lateral medullary syndrome may be a reversed pressure gradient caused by incoordination of pharyngeal contractility and UES opening during swallowing.

\section{Introduction}

Lateral medullary syndrome (LMS) is a neurological disease caused by the lesion of the lateral part of the medulla oblongata. ${ }^{1}$ Dysphagia, a common complication of LMS, is clinically important because of its associations with aspiration pneumonia, malnutrition, increased mortality, and decreased quality of life. Previous studies have suggested that the swallowing center, which includes the nucleus ambiguus (NA) and the nucleus tractus solitarius (NTS), coordinates the pharyngeal phases of swallowing. Fujishima et al. reported UES and pharyngeal incoordination during swallowing in dysphagia patients with LMS using conventional sensor. ${ }^{2}$ However, the pathophysiology of dysphagia has not been fully elucidated in these patietns. ${ }^{1,2}$

Herein, we present a case in which high-resolution manometry (HRM) was used to examine the pathophysiology of a patient with severe bulbar dysphagia due to LMS.

\section{Case Report}

A 41-year-old man presented with gait disorder, hoarseness, and dysphagia. He was diagnosed with a left LMS due to infarction (Fig. 1), and oral antiplatelet therapy was initiated. His neurological deficit almost improved except for severe dysphagia. Eleven months after the onset, he underwent a percutaneous endoscopic gastrostomy. Fourteen months after the onset, he was transferred to the rehabilitation department of our hospital for dysphagia treatment.

A videofluoroscopic examination of swallowing (VF) revealed weak pharyngeal contractility and an impaired upper esophageal sphincter (UES) function. The bolus remained in the pyriform sinus and did not pass through the pharynx (Fig. 2). The swallowing pressure along the pharynx and UES was measured using HRM. HRM revealed weak pharyngeal constriction and strong constriction of the UES muscle zone (Fig. $3)$. We used the pharyngeal contractile integral $(\mathrm{CI} ; \mathrm{mmHg} \cdot \mathrm{cm} \cdot \mathrm{s})$ to evaluate the pharyngeal swallowing pressure. ${ }^{3,4}$ The patient was examined for five dry swallows. During swallowing, the velopharyngeal CI (VPCI) and mesohypopharyngeal CI (MHPCI) values were $4.1 \pm 2.9$ and $72.0 \pm 16.2$ respectively (As reference values for this patient data, the VPCI and MHPCI values for $3 \mathrm{~mL}$ of thickened liquids in our previous report were $124.3 \pm 50.3$ and $193.2 \pm 34.1$, respectively ${ }^{4}$ ). Regarding the patient's UES function, the UESCI was $713.7 \pm 72.7$, indicating strong contraction during swallowing.

The patient received nutritional treatment, respiratory training, and swallowing rehabilitation (i.e., balloon catheter dilation, Shaker exercise, and vacuum swallowing therapies). He was discharged 16 months after the initial onset. He is continuing his swallowing rehabilitation at home and receiving treatment on an outpatient basis. This study was approved by the Ethics Committee of our hospital.

\section{Discussion}

To our knowledge, this was the first report to biomechanically clarify the pathophysiology of severe dysphagia due to LMS using HRM. The primary finding of this study was that the UES closed strongly during swallowing in a bulbar dysphagic patient with LMS. During normal swallowing, UES pressure drops to zero or below, whereas UES pressure in our patient increased abnormally during swallowing.

Notably, we calculated the pharyngeal CI as a measure of contractility to evaluate the pressure gradient between pharyngeal contraction and an impaired UES function. The CI was calculated as amplitude $\mathrm{x}$ duration $\mathrm{x}$ length of muscular contraction [?] $20 \mathrm{mmHg}$. It can act as a useful indicator of pharyngeal swallowing disorders. ${ }^{2}$ The CI is a measure of the "vigor" of contractility, and it has the potential to provide objective representations of pharyngeal and UES muscular functions. Compared with normal subjects, this patient's VPCI and MHPCI values were low owing to pharyngeal contractile weakness, whereas his UESCI was dramatically high owing to abnormal UES closing. The weakened pharyngeal contraction and 
dramatically closed UES during swallowing significantly interfere with the pharyngeal passage of a bolus in this patient with severe bulbar dysphagia.

HRM provided a more accurate and quantitative assessment of swallowing function. The high-pressure zone of the UES is narrow and asymmetric, and it moves up and down with the elevation of the larynx during swallowing. Therefore, a catheter with circumferential sensors is necessary to evaluate the UES function precisely. Decreased pharyngeal contraction and elevation of UES pressure during swallowing was reported using conventional sensors in these studies. ${ }^{2,5}$ It is difficult to precisely measure the "vigor" of abnormal UES contractility with these conventional sensors.

The central pattern generator $(\mathrm{CPG})$ for swallowing, located in the medulla, manipulates and controls the oropharyngeal phase of the swallowing sequence. ${ }^{1}$ Abnormalities in the UES function owing to dysphagiacausing LMS have been reported, but there have been few reports regarding the associated pathophysiology. The NA and NTS, located in the lateral medulla of the CPG, control the muscles of the pharynx and UES. Therefore, an LMS lesion that includes the NA and NTS can cause weak pharyngeal contraction and abnormal UES relaxation. This may lead to severe bolus residue in the pyriform sinuses after swallowing and, therefore, the possibility of subsequent aspiration. Patients with bulbar dysphagia due to lesions in LMS may experience deficits in pharyngeal and UES function, such as UES and pharyngeal incoordination, abnormal UES relaxation and forceful closure, and weak pharyngeal contraction.

Continuous swallowing rehabilitation (i.e., balloon catheter dilation and Shaker exercise), nutritional treatment, and respiratory muscle training would be beneficial for these patients with failed UES relaxation. If there is no sufficient improvement with these approaches, then clinicians could apply cricopharyngeal myotomy as an additional treatment. This is the only case report on this topic, which may be a limitation. Therefore, future studies should evaluate additional cases to verify our case findings.

\section{Conclusion}

One of the mechanisms of severe dysphagia due to lesions in the medulla oblongata may be a reversed pressure gradient caused by the incoordination of pharyngeal contractility and UES opening. Further studies should elucidate the pathophysiology, prognosis, and treatment of severe dysphagia due to LMS.

\section{Author's contributions}

Study concept and design : Kenjiro Kunieda, Takashi Shigematsu, Hideaki Kanazawa, Ichiro Fujishima

Acquisition of data : Kenjiro Kunieda, Takafumi Sugi, Akiko Nomoto

Analysis and interpretation of data : Kenjiro Kunieda, Tomohisa Ohno, Akiko Nomoto, Ichiro Fujishima

Drafting of the manuscript : Kenjiro Kunieda, Takafumi Sugi, Tomohisa Ohno, Akiko Nomoto, Takashi Shigematsu, Hideaki Kanazawa, Ichiro Fujishima

\section{References}

1. Jang SH, Kim MS. Dysphagia in lateral medullary syndrome: a narrative review. dysphagia. 2020. doi: $10.1007 / \mathrm{s} 00455-020-10158-3$

2. Fujishima I, Shibamoto I, Ohkuma R, Kojima C, Hojo K. Side of bolus passage at cricopharyngeal portion in Wallenberg's syndrome. Neuro Med. 2000. 52(3):309-15.

3. O'Rourke A, Humphries K, Lazar A. The pharyngeal contractile integral is a useful indicator of pharyngeal swallowing impairment. Neurogastroenterol Motil. 2017;29:e13144.

4. Kunieda K, Fujishima I, Wakabayashi H, et al. Relationship between tongue pressure and pharyngeal function assessed using high-resolution manometry in older dysphagia patients with sarcopenia: a pilot study. Dysphagia. 2020. doi:10.1007/s00455-020-10095-1. Online ahead of print.

5. Higo R, Tayama N, Watanabe T. Manometric abnormality in dysphagic patients after medullary cerebrovascular accidents. ORLJ Otorhinolaryngol Relat Spec. 2002;64:368-72.

\section{Figure Legends}


Figure 1. Magnetic resonance imaging 14 months after onset

T2-weighted image showing high intensity confined to a small region of the left dorsolateral medulla (yellow arrow).

Figure 2. Videofluoroscopic examination of swallowing 14 months after onset

Severe bolus residue in the pyriform sinus owing to weak pharyngeal contraction and an impaired UES relaxation.

Figure 3. Pressure topographies of the patient (a) and of a normal subject (b)

Spatiotemporal plots of dry swallows. Y-axis, catheter position; X-axis, time. Pressure is indicated using the color scale. Maximum pharyngeal pressures measured at the velopharynx and meso-hypo pharynx. UES relaxation duration and UES pressure during swallowing are evaluated.

UES: upper esophageal sphincter.

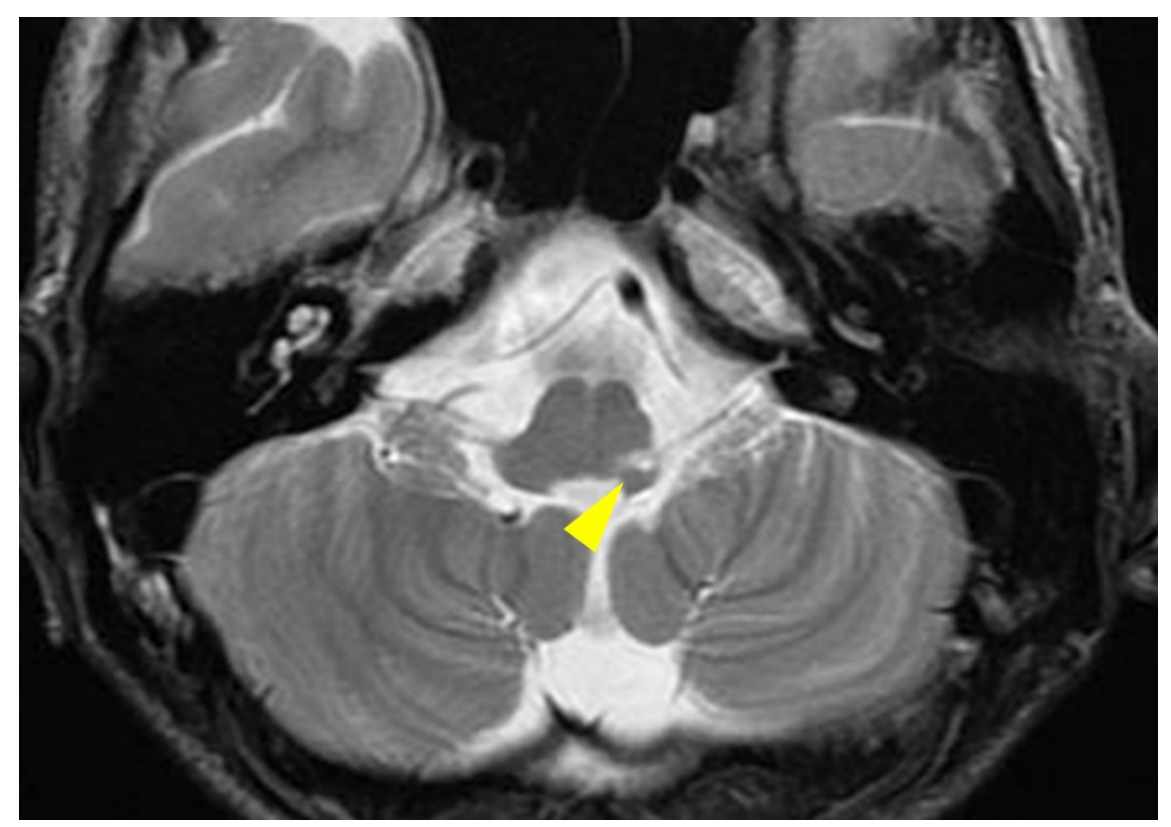



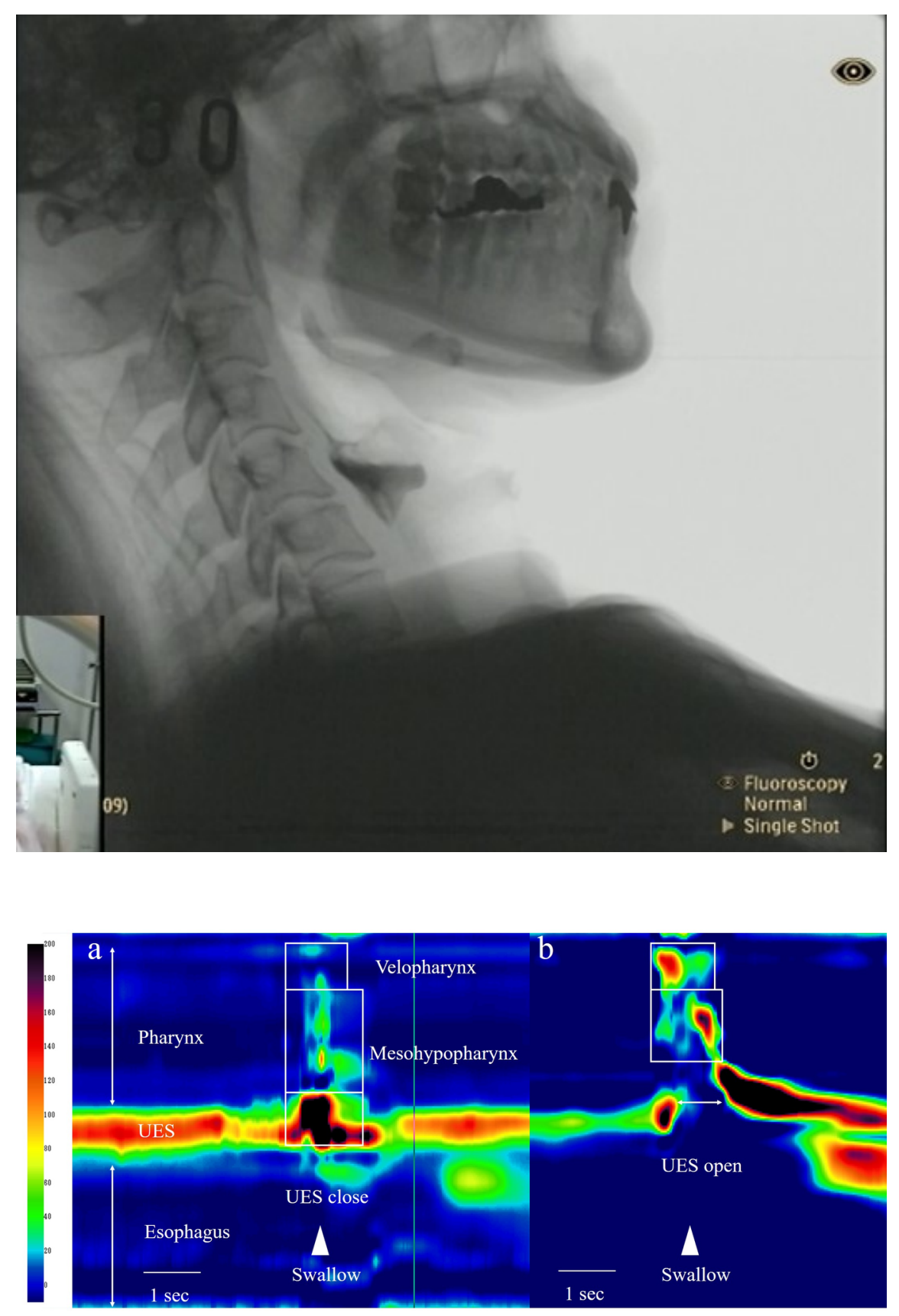